\title{
Costos directos del glaucoma primario de ángulo abierto
}

\section{Direct costs of open angle primary glaucoma}

\author{
Dra. Ariana Fernández García,' Dra. I dalia Triana Casado," DrC. Rosendo \\ Villar Valdés'"' \\ ' Escuela Nacional de Salud Pública. La Habana, Cuba. \\ "Hospital "Dr. Salvador Allende". La Habana, Cuba. \\ II' Facultad de Ciencias Médicas "Calixto García". La Habana, Cuba.
}

\section{RESUMEN}

I ntroducción: la magnitud del costo asociado al glaucoma primario de ángulo abierto deriva de la atención sanitaria y de la disminución de la calidad de vida del paciente. Objetivos: estimar los costos del glaucoma primario de ángulo abierto en los pacientes atendidos durante el 2010 en el servicio de glaucoma del hospital "Dr. Salvador Allende."

Métodos: se realizó un estudio retrospectivo, en el campo de la Evaluación Económica en Salud es un estudio del tipo de descripción de costos, que aplicó el enfoque metodológico del costo de la enfermedad. El universo de estudio estuvo constituido por el total de casos con diagnóstico de glaucoma primario de ángulo abierto que acudieron a la consuñlta seleccionada de glaucoma del citado hospital, durante el primer trimestre del 2011 y que habían sido atendidos durante el 2010. Los costos fueron expresados en pesos cubanos del 2010. El estudio se realizó a partir de las perspectivas de la institución y la del paciente.

Resultados: el estudio incluyó 110 pacientes con edad promedio de 60,3 años de edad. El costo promedio de la atención a un paciente en la institución fue de 230,99 pesos cubanos. El costo promedio para los pacientes fue de 290,10 pesos cubanos. Conclusiones: el servicio de glaucoma cuenta con los recursos científico técnicos imprescindibles para la atención de los pacientes. Los costos de la enfermedad para el paciente son cubiertos con los ingresos monetarios que estos perciben.

Palabras clave: glaucoma primario de ángulo abierto, costo de la enfermedad, economía de la salud. 


\section{ABSTRACT}

Introduction: the magnitude of the cost of open angle primary glaucoma stems from the health care and the reduction of the patient's quality of life.

Objectives: to estimate the open angle primary glaucoma costs in those patients seen at the glaucoma service of "Salvador Allende" hospital during 2010.

Methods: a retrospective cost description-type study, which applied the methodological approach to the disease costs, was carried out in the field of health economic assessment. The universe of study was the total number of cases diagnosed as open angle primary glaucoma and seen at the glaucoma service during the first quarter of 2011 and those which had been treated during 2010. The costs were given in Cuban pesos, according to the 2010 rate. The study was conducted on the basis of the perspectives of the institution and those of the patients.

Results: the study comprised 110 patients aged 60.3 years. The average cost of assistance to a patient at the hospital was 230.99 Cuban pesos. The average cost for the patients was 290.10 Cuban pesos in the analyzed period.

Conclusions: the glaucoma service has the indispensable scientific and technical resources for the care of patients. The costs of the disease for the patients are afforded by their own incomes.

Key words: open angle primary glaucoma, cost of disease, health economics.

\section{NTRODUCCI ÓN}

El glaucoma constituye una causa importante de ceguera irreversible a nivel mundial. El glaucoma primario de ángulo abierto (GPAA), o glaucoma crónico simple, es el más frecuente de todos los glaucomas, se caracteriza por ausencia de causas secundarias y los ángulos iridocorneales aparecen abiertos, a menudo es hereditario, y probablemente intervienen varios factores.

La incidencia a nivel mundial del GPAA tiene una amplia variación racial y étnica. En general, su incidencia puede ser de 1 a $3 \%$, y se incrementa a medida que aumenta la edad de los pacientes, por ejemplo, llega al $5 \%$ en las personas de 75 años. ${ }^{1}$

EI GPAA, es una enfermedad crónica con instauración insidiosa que, con frecuencia, no se detecta sino hasta que se produce una pérdida considerable del campo visual. Aunque la enfermedad es casi siempre bilateral, la progresión es a menudo asimétrica, de modo que el paciente con frecuencia se presenta con pérdida significativa del campo visual en un ojo y con la enfermedad menos avanzada en el otro. En ocasiones los pacientes con presión intraocular elevada pueden quejarse de dolor en los ojos, cefalea e incluso de halos causados por un edema transitorio del epitelio de la córnea. ${ }^{1,2}$

Tanto la enfermedad como la discapacidad a que conlleva el GPAA tienen impacto sobre la vida cotidiana de los enfermos y sobre la de sus familiares. Así, los pacientes con glaucoma pueden perder calidad de vida por varias razones, como son: el diagnóstico por sí mismo, que puede causar angustia; la pérdida insidiosa de la independencia y de la visión, que resulta en una reducción de las actividades diarias y disminución de la confianza para realizar actividades al aire libre; los inconvenientes 
del tratamiento, por la frecuencia de aplicación y el seguimiento regular por consulta externa en los hospitales y los efectos secundarios del tratamiento y sus costos. ${ }^{3}$

En Cuba, el GPAA constituye un importante problema de salud. ${ }^{4-6}$ En una investigación realizada en el 2006 en el municipio Las Tunas, donde está concentrada la mayor densidad poblacional de la provincia, se encuentra una incidencia de GPAA de 3,7 \% en el grupo de los mayores de 40 años de edad que ascendió a 6,6 \% en el grupo de 60 años en adelante. ${ }^{5}$

Se han realizado varios estudios que estiman el costo de la enfermedad en el contexto del Sistema de Salud cubano, ${ }^{7-11}$ en general, el propósito de estos ha sido aportar información para tomar decisiones y elevar la eficiencia de los servicios. Sin embargo, no aparecen referencias en la búsqueda bibliográfica realizada en el marco de este trabajo, de investigaciones que determinen el costo de la enfermedad en oftalmología.

El objetivo de este trabajo es estimar los costos del glaucoma primario de ángulo abierto en los pacientes atendidos durante el 2010 en el servicio de glaucoma del hospital "Dr. Salvador Allende."

\section{MÉTODOS}

Se realizó un estudio retrospectivo, en el campo de la Evaluación Económica en Salud es un estudio parcial del tipo de descripción de costos, que aplicó el enfoque metodológico del costo de la enfermedad y empleó la Guía Metodológica para las Evaluaciones Económicas en Salud en Cuba. ${ }^{12}$ Se tuvieron en cuenta los costos ocurridos en la atención y el tratamiento de los pacientes durante el 2010. Se utilizaron los costos clasificados como costos directos sanitarios y no sanitarios, costos fijos, costos variables, costos totales, y costos promedio, expresados en pesos cubanos del 2010.

El estudio se realizó a partir de las perspectivas de la institución y la del paciente. De tal manera, se registraron aquellos costos que se relacionaban con los servicios de salud prestados por el sistema en cuestión y los que se correspondían con los gastos de bolsillo del paciente. Se basó en el enfoque de la prevalencia de la enfermedad y se combinaron técnicas cuantitativas y cualitativas.

El universo de estudio estuvo constituido por el total de casos con diagnóstico de GPAA, que acudieron a la consulta seleccionada del servicio de glaucoma del hospital "Dr. Salvador Allende" de La Habana durante el primer trimestre del 2011 y que fueron atendidos durante el 2010.

Se incluyeron los pacientes con el diagnóstico confirmado de GPAA, realizado previamente a su inserción en el estudio por el médico especialista de la consulta seleccionada, con un año o más de tratamiento de la enfermedad; mayores de 18 años de edad, que se encontraban asistiendo regularmente a la consulta. En el diagnóstico del paciente se consideró la exploración oftalmológica, la presión intraocular ajustada por paquimetría, gonioscopia según el sistema de Shaffer, evaluación del nervio óptico y capa de fibras nerviosas de la retina, estudio del campo visual y agudeza visual mejor corregida.

Los criterios de exclusión fueron los pacientes con enfermedad ocular asociada que pudiera provocar defectos campimétricos y visuales profundos como desprendimiento 
de retina, degeneración macular, catarata o retinopatía diabética, aquellos en los que se manifestó algún tipo de confusión o imposibilidad de comprender el cuestionario y los que no dieron su consentimiento para participar.

En los costos directos sanitarios se consideraron los costos para la institución.

Las variables analizadas fueron: costos por recurso humano, por consultas, por estudios diagnósticos y por otros servicios generales. En general, se aplicó igual procedimiento que el aplicado en estudios anteriores de costo institucional. ${ }^{7,10}$

El costo de los recursos humanos se calculó a partir del salario integrado neto del personal que participa directa e indirectamente en la atención al paciente. Se determinó el número de profesionales relacionados con la atención de los pacientes con GPAA, su salario y el tiempo en horas y minutos dedicados a la atención de un paciente, mediante entrevista no estructurada y personal, revisión de nóminas y comprobación por el documento SNS-225. Se tuvo en cuenta para la estimación las 190,6 h (indicador de tiempo mensual según la legislación laboral de Cuba), la contribución a la seguridad social $(12,5 \%)$, el descanso retribuido acumulado $(9,09 \%)$ y la fuerza de trabajo $(25,0 \%)$.

En el costo por consultas y por estudios diagnósticos se revisó la historia clínica ambulatoria y la historia clínica interna del hospital de cada paciente, y se consideró la tarifa de los procederes técnicos que se aplican en los centros de salud, para lo cual se consultó a funcionarios del área económica del Ministerio de Salud Pública. Los procederes incluidos fueron: fondo de ojo, gonioscopia, determinación de la presión intraocular, estudio de la agudeza visual y del campo visual.

El costo por otros servicios generales incluyó: el agua, electricidad, teléfono, y otros servicios prestados.

Para la estimación de las partidas se hicieron consultas a documentos oficiales del servicio de glaucoma del hospital y consultas a expertos en almacén general y de medicamentos, departamentos de estadística y contabilidad, médicos, enfermeras y tecnólogos que atienden directamente a estos pacientes y encargados de la toma de decisiones. Es de señalar que, en esta estimación de costo, no se consideró el costo por cirugía, ni por hospitalización, por que no fueron necesarias en ninguno de los pacientes incluidos.

En los costos directos no sanitarios se consideraron los costos para el paciente. Se confeccionó un cuestionario ajustado a estos efectos, previa consulta del cuestionario utilizado por Sanabria y otros, ${ }^{13}$ y el confeccionado por Yera Alós. ${ }^{14}$ El cuestionario fue aplicado por una de las autoras mediante entrevista personal, de manera que se aclararon las dudas del paciente al momento de su completamiento. Para este propósito se incluyeron las variables: situación laboral, nivel de ingresos monetarios, tipo de medio de transporte, costo del transporte, costo de merienda, costo del dispositivo de baja visión y costo de medicamentos. En el costo por medicamentos se consideró el precio unitario establecido a nivel nacional. No fueron incluidos los medicamentos que la institución adquiere por concepto de donación y que el paciente recibe sin costo alguno. Se realizó la sumatoria del costo total de cada una de las partidas y se dividió entre el número de casos para obtener el costo promedio por paciente.

En cada caso, para el cálculo del costo total se utilizó el enfoque de abajo hacia arriba, que cuantifica los recursos de forma desagregada para cada paciente, ya que se consideró que el uso de los recursos puede ser variable en función de este. Se realizó la sumatoria de los costos totales de cada paciente, se calculó el costo 
promedio de atención de la enfermedad y se multiplicó por el total de casos estudiados. Los datos se analizaron mediante indicadores descriptivos.

La Comisión de Ética del hospital "Dr. Salvador Allende", y los responsables de los departamentos donde se procesaron primariamente los datos, dieron su aprobación para la toma de la información necesaria para la realización del estudio; se tuvo en cuenta su confidencialidad.

\section{RESULTADOS}

El estudio incluyó 110 pacientes, de los cuales 79 (71,81\%) fueron del sexo femenino. La edad promedio fue de 60,3 años de edad (variando entre 19 y 93), con mayor frecuencia de pacientes entre 60 y 69 años de edad (tabla 1).

Tabla 1. Distribución de pacientes con glaucoma primario de ángulo abierto según edad

\begin{tabular}{|l|r|r|}
\hline $\begin{array}{l}\text { Grupos } \\
\text { de edad (años) }\end{array}$ & No. & \multicolumn{1}{c|}{$\%$} \\
\hline Menos de 20 & 2 & 1,81 \\
\hline 20 a 29 & 2 & 1,81 \\
\hline 30 a 39 & 4 & 3,64 \\
\hline 40 a 49 & 14 & 12,73 \\
\hline 50 a 59 & 27 & 24,55 \\
\hline 60 a 69 & 31 & 28,18 \\
\hline 70 a 79 & 25 & 22,73 \\
\hline 80 a 89 & 4 & 3,64 \\
\hline Más de 89 & 1 & 0,91 \\
\hline Total & 110 & 100,00 \\
\hline
\end{tabular}

El tiempo promedio de diagnosticado el GPAA fue de 10,3 años. De los pacientes estudiados, 55 tenían más de 10 años de diagnosticada la enfermedad (50,0\%), 34 pacientes tenían un tiempo entre 10 y 5 años (30,91 \% ) y 21 pacientes tenían menos de 5 años $(19,09 \%)$.

El número de consultas promedio en los pacientes del estudio fue de 3,79 en el año. El costo promedio anual de la atención a un paciente con GPAA en la institución fue de 230,99 pesos cubanos, que osciló en el rango de 152,79 hasta 448,89 pesos cubanos (IC $95 \%$ : 174,02-287,96), y un costo total anual de 25408,50 pesos cubanos en el año 2010 para la atención de los 110 pacientes estudiados (tabla 2).

Del total de pacientes, solo $8(7,27 \%)$ habían necesitado tratamiento quirúrgico para el control de la enfermedad, pero antes del período de estudio.

De los pacientes estudiados, $106(96,36 \%)$ estaban utilizando tratamiento farmacológico (tabla 3 ), seis de estos pacientes adquirían el medicamento gratuitamente en el hospital y tres lo recibían de sus familiares. 
Tabla 2. Costo anual de la atención al paciente con glaucoma primario de ángulo abierto

\begin{tabular}{|c|c|c|c|c|}
\hline Concepto & Costo total & $\mathrm{n}$ & $\begin{array}{c}\text { Costo } \\
\text { promedio }\end{array}$ & $\%$ \\
\hline Estudios diagnósticos & 12804,00 & 110 & 116,40 & 50,39 \\
\hline Consultas de oftalmología & 8962,80 & 110 & 81,48 & 35,28 \\
\hline Recursos humanos & 2823,70 & 110 & 25,67 & 11,11 \\
\hline Otros costos & 818,40 & 110 & 7,44 & 3,22 \\
\hline Total & 25408,50 & 110 & 230,99 & 100,00 \\
\hline
\end{tabular}

Tabla 3. Distribución de los pacientes con glaucoma primario de ángulo abierto según el fármaco antiglaucomatoso que utilizan

\begin{tabular}{|l|r|r|}
\hline Fármaco & No. & \multicolumn{1}{|c|}{$\%$} \\
\hline Timolol & 71 & 66,98 \\
\hline Pilocarpina & 15 & 14,15 \\
\hline Latanoprost & 6 & 5,66 \\
\hline Dorzolamida & 5 & 4,71 \\
\hline Timolol + pilocarpina & 4 & 3,77 \\
\hline Timolol + latanoprost & 2 & 1,88 \\
\hline Timolol + brimonidina & 1 & 0,95 \\
\hline Unoprostona & 1 & 0,95 \\
\hline Latanoprost + dorzolamida & 1 & 0,95 \\
\hline Total & 106 & 100,00 \\
\hline
\end{tabular}

En relación con la ocupación, 54 pacientes eran jubilados $(49,09 \%), 39$ eran trabajadores estatales (35,45\%), 12 amas de casa (10,91\%), 3 estudiantes $(2,73 \%)$ y 2 trabajadores por cuenta propia (1,82 \%). La pensión promedio mensual de los pacientes jubilados era de 264,34 pesos cubanos para un promedio anual de 3172,08 pesos cubanos, y el salario promedio mensual de los trabajadores estatales era de 460,20 pesos cubanos para un promedio anual de 5522,40 pesos cubanos.

De los pacientes del estudio, 66 viven en áreas cercanas al hospital y acuden a la consulta caminando

$(60,00 \%)$, el resto utilizó los siguientes medios: 23 pacientes transporte público $(20,91 \%), 9$ pacientes transporte particular $(8,18 \%), 7$ pacientes transporte estatal $(6,36 \%)$ y 5 pacientes transporte de alquiler $(4,55 \%)$.

Solo 9 pacientes $(8,18 \%)$ refirieron necesitar ayuda a causa de la disminución visual para realizar actividades dentro o fuera del hogar y $96(87,27 \%)$ tenía comorbilidades sistémicas, que no causan daño ocular o que no mostraban repercusión ocular.

El costo promedio anual del GPAA para el paciente en el período analizado fue de 290,10 pesos cubanos, y se encontró en un rango amplio de 59,00 hasta 5 099,00 pesos cubanos (tabla 4).

http://scielo.sld.cu 
Tabla 4. Costo promedio anual del glaucoma primario de ángulo abierto para el paciente

\begin{tabular}{|l|c|c|}
\hline Concepto & $\begin{array}{c}\text { Costo } \\
\text { promedio }\end{array}$ & $\%$ \\
\hline Medicamentos & 135,49 & 46,70 \\
\hline Asistir a consultas & 95,18 & 32,81 \\
\hline Espejuelos & 59,43 & 20,49 \\
\hline Total & 290,10 & 100,00 \\
\hline
\end{tabular}

\section{DISCUSIÓN}

El costo total anual de la atención institucional a los 110 pacientes con GPAA incluidos en el estudio fue de 25 408,50 pesos cubanos, que representó el 0,09\% del presupuesto anual del hospital "Dr. Salvador Allende" del 2010 que fue de 26 millones de pesos cubanos. La mitad de los costos institucionales están dados por los estudios diagnósticos que permiten establecer el nivel de progresión de la enfermedad. El costo institucional se incrementó fundamentalmente por el número de consultas de seguimiento que requiere el paciente en función de la evolución de la enfermedad.

El costo promedio anual del GPAA para el paciente fue de 290,10 pesos cubanos, que representó el 9,14 \% del ingreso promedio anual de los jubilados ( 3172,08 pesos cubanos) por sus pensiones y el 5,25\% del ingreso promedio anual de los trabajadores estatales ( 522,40 pesos cubanos), por sus salarios. Cerca de la mitad de los costos para el paciente estuvieron relacionados con los medicamentos indicados. El costo para el paciente se incrementó a partir de la utilización de transporte de alquiler para acudir a consulta y, en menor medida, por adquirir medicamentos en las farmacias internacionales del país cuando existen contraindicaciones para el uso de los fármacos disponibles en las farmacias comunitarias.

El costo de los medicamentos resultó accesible a todos los pacientes. De los pacientes que tenían que comprar su tratamiento solo uno tuvo que adquirirlo en farmacia internacional por un corto período de tiempo, de manera que casi la totalidad de los pacientes adquirieron en las farmacias comunitarias sus medicamentos donde el precio está subvencionado por el Estado.

No se incluyó el costo de los medicamentos de los tres pacientes que los recibían de sus familiares, porque no fue posible obtener esta información. Además, se debe considerar que un grupo elevado de los pacientes estudiados tenía comorbilidades que requirieron tratamientos e incrementaron los gastos de bolsillo, pero que no constituyó objeto de este estudio.

En la literatura internacional existen referencias sobre el costo directo del glaucoma primario de ángulo abierto. Así, en el trabajo sobre costos institucionales del glaucoma y la hipertensión ocular realizado por Daniela Koleva y otros, ${ }^{15}$ el número promedio de consultas anuales en los pacientes con glaucoma fue de 3,68. Los autores refieren un costo promedio por consulta de oftalmología de 206,4 EUR y un costo promedio por pruebas diagnósticas de 99,6 EUR, a los que se adicionan los costos por medicamentos, cirugía y hospitalización para un costo promedio anual de atención al paciente de 734,3 EUR. 
Un estudio sobre el costo del glaucoma para el sistema de salud en dólares estadounidenses en Estados Unidos, estimó un total de \$2,5 mil millones anuales; de los cuales $\$ 1,9$ mil millones constituyeron costos directos y $\$ 0,6$ mil millones costos indirectos. ${ }^{16}$

Las investigaciones sobre las implicaciones sociales y económicas del glaucoma, refieren que los costos de la atención al paciente con esta enfermedad aumentan a medida que empeora la enfermedad y que la efectividad en el manejo del paciente y el retraso en la progresión de la enfermedad pueden reducir significativamente su carga económica. Además, resaltan la necesidad de realizar estudios enfocados en la perspectiva social para respaldar sus conclusiones. ${ }^{16-20}$

En un estudio del 2006, realizado en Estados Unidos sobre la carga económica total anual de los trastornos visuales más importantes en los adultos, se plantea que el costo económico en dólares estadounidenses ascendió a \$35,4 mil millones: $\$ 16,2$ mil millones en costos médicos directos, $\$ 11,1$ mil millones en otros costos directos y $\$ 8$ mil millones en pérdidas de productividad. En particular, los gastos médicos directos para cada una de las afecciones estudiadas fueron: $\$ 6,8$ mil millones para la catarata, $\$ 5,5$ mil millones para los trastornos refractivos, $\$ 2,9$ mil millones para el glaucoma, \$575 millones para la degeneración macular asociada a la edad y $\$ 493$ millones para la retinopatía diabética. ${ }^{17}$ Estos resultados hacen reflexionar sobre la necesidad de realizar estudios de costo de la enfermedad en el contexto de Cuba, no solo del GPAA, sino también de otros trastornos visuales que afectan a la población cubana.

Este estudio constituye una aproximación al tema del costo del GPAA en Cuba, que se continuará trabajando como parte del proyecto "Salud visual y costos del glaucoma primario de ángulo abierto. Metodología en el contexto cubano."

Si bien el GPAA es una enfermedad costosa, el Estado cubano no ha limitado los recursos materiales, humanos y financieros necesarios según el desarrollo científico técnico de la Oftalmología. El servicio de glaucoma del hospital "Dr. Salvador Allende" cuenta con los recursos imprescindibles para la atención de estos pacientes. Los costos de la enfermedad para el paciente son cubiertos con los ingresos monetarios que estos perciben.

\section{Agradecimientos}

Se hace un reconocimiento a todos los médicos, enfermeras y otros profesionales del servicio de glaucoma del hospital "Dr. Salvador Allende" que permitieron que esta investigación se pudiera realizar.

\section{REFERENCI AS BI BLI OGRÁFICAS}

1. Duane's Ophthalmology. Tasman W, Jaeger EA, editors. Philadelphia: Lippincott Williams \& Wilkins; 2007.

2. Ravi T, Loibl K, I Parikh R. Evaluation of a glaucoma patient. Indian J Ophthalmol. 2011;59(Suppl 1):S43-S52. 
3. Severn P, Fraser S, Finch T, May C. Which quality of life score is best for glaucoma patients and why? BMC Ophthalmology [Internet]. 2008 [cited 2008 Feb 4 ]; 8(2).

Disponible en: http://www.biomedcentral.com/1471-2415/8/2

4. Prats Aleaga VC, Rondón Paz N, Pérez Viladeba L. Pesquisaje oftalmológico. Rev Cubana Oftalmol [Internet]. 1995 [citado 6 Abr 2011];8(1). Disponible en: http://bvs.sld.cu/revistas/oft/vol8_1_95/oft08195.htm

5. Labrada Rodríguez Y, Hornia Palacios M, González Hess L, Rodríguez Suárez J. Glaucoma primario de ángulo abierto. Un problema de salud en el municipio Las Tunas. Rev Cubana Oftalmol [internet]. 2006 [citado 6 Abr 2011]; 19(1). Disponible en: http://scielo.sld.cu/scielo.php?script=sci_arttext\&pid=S0864 -

$21762006000100005 \&$ lng $=e s \& n r m=i s o \& t \mid n g=e s$

6. Pérez Díaz L. Glaucoma: principal problema de salud en los miembros de la Asociación Nacional del Ciego en Santiago de Cuba. MEDISAN [Internet]. 2009 [citado 4 de junio de 2011]; 13(2). Disponible en: http://bvs.sld.cu/revistas/san/vol13_2_09/san05209.htm

7. Fernández García A, Gálvez González AM, Castillo Guzmán A. Costo institucional del Infarto Agudo del Miocardio. Instituto de Cardiología y Cirugía Cardiovascular. La Habana. 2006. Rev Cubana Salud Pública [Internet]. 2008 [citado 4 de junio de 2011];34(4). Disponible en:

http://bvs.sld.cu/revistas/spu/vol34_4_08/spu06408.htm

8. Sarria GD, Espinosa BA, Hidalgo PF. Costos de la atención a pacientes en la clínica del diabético de Cienfuegos. Año 2006 [tesis]. La Habana: Escuela Nacional de Salud Pública; 2008.

9. Nicolás PR, Espinosa BA. Costo de la atención médica a pacientes hipertensos. Policlínico Aracelio Rodríguez Castellón de Cumanayagua. Año 2006 [tesis]. La Habana: Escuela Nacional de Salud Pública; 2008.

10. Tabares Silverio Y, Fernández García A, Castillo Abreu D. Costo Institucional de la Crisis Hipertensiva. Policlínico Antonio Maceo. Rev Cubana Med Gen Integr [internet]. 2011 J un [citado 14 de julio de 2011];27(2). Disponible en: http://bvs.sld.cu/revistas/mgi/vol27_02_11/mgi04211.htm

11. Lam Díaz RM, Gálvez González AM, Hernández Ramírez P. Repercusión económica del tratamiento de la leucemia promielocítica. Experiencia en el Instituto de hematología. La Habana. 2001-2006. INFODIR [internet]. 2010 [citado 4 de junio de 2011]; 10. Disponible en: http://bvs. sld.cu/revistas/infd/n1010/infd0410.htm

12. Gálvez González AM. Guía metodológica para la evaluación económica en salud: Cuba, 2003. Rev Cubana Salud Pública [Internet]. 2004 Mar [citado 4 de junio de 2011];30(1). Disponible en:

http://scielo.sld.cu/scielo.php?script=sci_arttext\&pid=S0864-

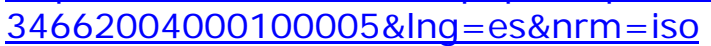

13. Sanabria G, Gálvez AM, Álvarez M. Costo para la mujer durante la atención prenatal. Ciudad de La Habana. 2000. Rev Cubana Salud Pública. 2004;30(1): 77-82.

14. Yera Alós I. Gasto de bolsillo de pacientes con cáncer en una sesión de quimioterapia ambulatoria. INOR. Mayo. 2007 [tesis]. La Habana: Escuela Nacional de Salud Pública; 2008.

http://scielo.sld.cu 
15. Koleva D, Motterlini I, Schiavone M, Garattini L. Medical costs of glaucoma and ocular hypertension in I talian referral centres: a prospective study. Ophthalmologica. 2007; 221:340-7.

16. Lee P, Walt J, Doyle J, Kotak S, Evans S, Budenz D, et al. A multicenter, retrospective pilot study of resource. Use and costs associated with severity of disease in glaucoma. Arch Ophthalmol. 2006; 124: 12-9.

17. Rein D, Ping Zhang, Wirth K, Lee $\mathrm{P}$, Hoerger $\mathrm{T}$, et al. The Economic Burden of Major Adult Visual Disorders in the United States. Arch Ophthalmol. 2006; 124:175460.

18. Traverso CE, Walt J G, Kelly SP, Hommer AH, Bron AH, Denis P, et al. Direct costs of glaucoma and severity of the disease: a multinational long term study of resource utilisation in Europe. Br J Ophthalmol. 2005; 9(10): 1245-9.

19. Roberts C, Hiratsuka Y, Yamada M, Pezzullo L, Yates K, Takano S, et al. Economic cost of visual impairment in Japan. Arch Ophthalmol. 2010;128(6):766-71.

20. Bramley T, Peeples P, Walt JG, J uhasz M, Hansen JE. Impact of vision loss on costs and outcomes in medicare beneficiaries with glaucoma. Arch Ophthalmol. $2008 ; 126(6): 849-56$.

Recibido: 22 de mayo de 2012.

Aprobado: 29 de junio de 2012.

Ariana Fernández García. Escuela Nacional de Salud Pública. Calle 100 No. 1132 e/ E y Perla. Altahabana, municipio Boyeros. La Habana, Cuba. Correo electrónico:

ariana.fdez@infomed.sld.cu 